Review

\title{
Mechatronics Around the World - at a Glance
}

\author{
${ }^{1}$ Rachita Sharma and ${ }^{2}$ Bhaskar Dhiman \\ ${ }^{I}$ Department of Electronics and Communication Engineering, Abhilashi University, Mandi, H.P. India \\ ${ }^{2}$ Department of Mechanical Engineering, Abhilashi University, Mandi, H.P. India
}

\author{
Article history \\ Received: 03-11-2020 \\ Revised: 06-01-2021 \\ Accepted: 14-01-2021 \\ Corresponding Author: \\ Rachita Sharma \\ Department of Electronics and \\ Communication Engineering, \\ Abhilashi University, Mandi, \\ H.P. India \\ Email: rachitasharma13@gmail.com
}

\begin{abstract}
Increasing performance standards for complex procedures, which includes business equipment and their steadily growing technical importance, might require new processes to be applied within the procedure of product advent. The important thing concept in the back of our proposed approach is to combine one of a kind stages consisting of design, simulation and physical implementations in product or device advent. The distinguishing characteristic of mechatronic structures is illustrated in this study by using intensive integration. The mechatronic model allows us to better understanding the additive's complex behavior and interactions. This affords accelerated opportunities for the dynamic movement performance of the entire device to be measured and optimized inside the early levels of the layout process. The developing effect of interactions among machine additives on attainable gadget dynamics and accuracy is every other consequence. In this study, a concept for mechatronics is proposed to satisfy these requirements with the help of multiple diagrams and various applications.
\end{abstract}

Keywords: Mechatronics, Mechanical, Electronics, Electrical, Intensive Integration, Applications of Mechatronics, Actuators, Additives

\section{Introduction}

As generation progresses through the years, exceptional engineering subfields have succeeded in both adapting and multiplying. Mechatronics aims to produce a solution for layout that unifies each of these distinctive subfields. The mechatronics location became first of all supposed to be nothing extra than a combination of mechanics and electronics (Igweonu et al., 2013). Laptop engineering, manipulate engineering, systems engineering and telecommunication engineering are also covered. It is essentially the application of various technical fields to discover greater dependable solutions for product design and manufacturing. Tetsuro Mori, a Japanese engineer, first coined the term mechatronics in the late 1960s (Milecki, 2015).

It is mentioned that for researchers, mechatronics is not just a commodious shape; it is the manner of lifestyles in the current engineering generation. A traditional mechanical design technique and mechatronics design approach is shown in Fig. 1a and $1 \mathrm{~b}$.

In the case of the conventional layout technique, there is no integration with electronics engineering, computer engineering, IT engineering and control engineering. However, the mechatronics design method includes the integration of mechanical engineering, electronics, computer and IT engineering.

\section{Elements of Mechatronics}

Various elements in ordinary mechatronic buildings are demonstrated in Fig. 2 and are described proper right here below (Mishra et al., 2011):

a) Mechanical: Mechanical elements refer to the mechanical shape of a mechatronics device, mechanism, thermo-fluid and hydraulic components. Static/dynamic traits may be covered within the mechanical detail and it interacts purposefully with its environment. Bodily energy is needed through the mechanical additives of mechatronic systems to supply motion, force and heat and so on.

b) Electromechanical elements: Sensors and actuators confer with electromechanical factors. Sensors may be used to degree a diffusion of physical variables, e.g., Mild the usage of a 
photograph-resistor, stage and displacement using a potentiometer, direction/tilt the use of a magnetic sensor, microphone sound, pressure gauge stress; micro-transfer touch; thermistor temperature and conductivity sensor humidity. The physical method is controlled by actuators consisting of the Light-Emitting Diode (LED), DC servomotor, stepper motor, relay, solenoid, speaker, form reminiscence alloy, electromagnet and pump. IC-primarily based sensing and actuation solutions (e.g., Digital-compass, potentiometer and many others.) have additionally come to be ubiquitous in the latest years.

c) Electrical/Electronics: Electric factors consult with electrical components, circuits and analog indicators (e.g., Resistor (R), Capacitor (C), Inductor (I), Transformer, etc.). Analog/virtual electronics, transistors, thyristors, opto-isolators, operational amplifiers, strength electronics and signal conditioning consult with electronic factors. The electrical/electronic factors are used to interface the hardware elements of the management interface with electro-mechanical sensors and actuators.

d) Control interface/Computing hardware: Analog-tovirtual converters, a digital-to-analog converter, virtual enter/output $(\mathrm{I} / \mathrm{O})$, counters, timers, microprocessors, microcontrollers, fact Acquisition and manage (DAC) forums and virtual Sign Processing (DSP) forums are known as manage interface/computing hardware elements. The hardware of the management interface enables analog/digital interfacing, i.e., the communiqué

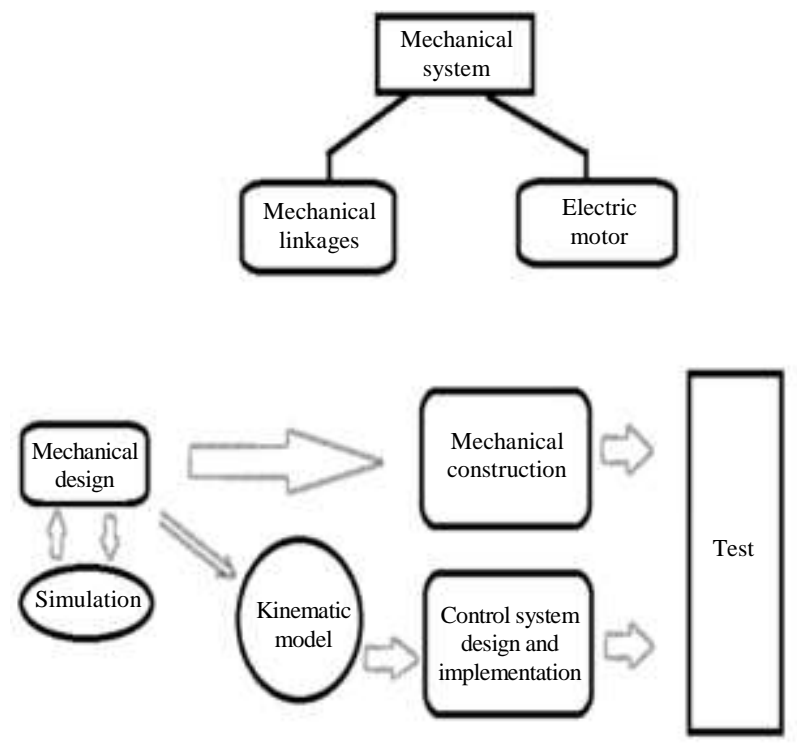

(a) of the sensor signs to the manipulate system and the verbal exchange of the manage sign to the actuator from the managed device.

e) Computer: Computer factors talk over with hardware/software program used for laptop-aided dynamic device analysis, optimization, layout and simulation; digital instrumentation; prototyping of fast manipulate; simulation of hardware-in-theloop; and acquisition and manipulate of laptopprimarily based data.

\section{The Value of Mechatronics}

Some of its most notable uses and advantages are provided below (Diegel et al., 2008):

a) Save time: In contrast to a machine with various engineering ideas detached from every different, an incorporated manufacturing line is lots quicker in operation. In contrast to doing it in exclusive phases and locations, it saves time while products are imaged, sorted, measured and recorded as they pass in a belt.

b) Increase in the output: A fast system way a terrific growth in output capability. Mechatronics has made it smooth to supply massive portions of merchandise.

c) Cost-effective: It means that you have less manpower used when you have an automated production line at work. In terms of labor costs, that means savings. Besides, an automated system is more operationally efficient, with fewer technical and input errors.

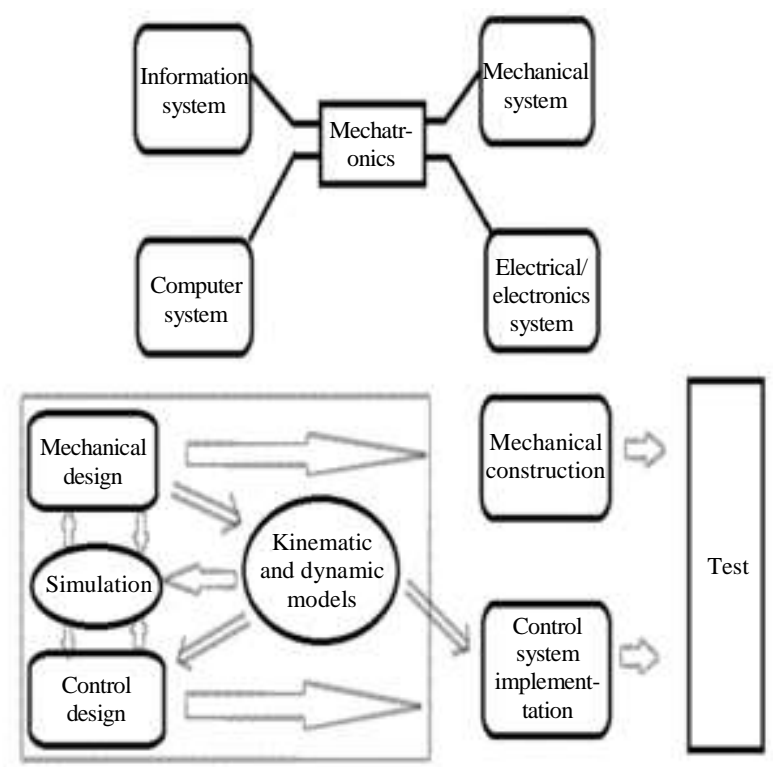

(b)

Fig. 1: (a) Traditional approach; (b) integrated mechatronics approach 


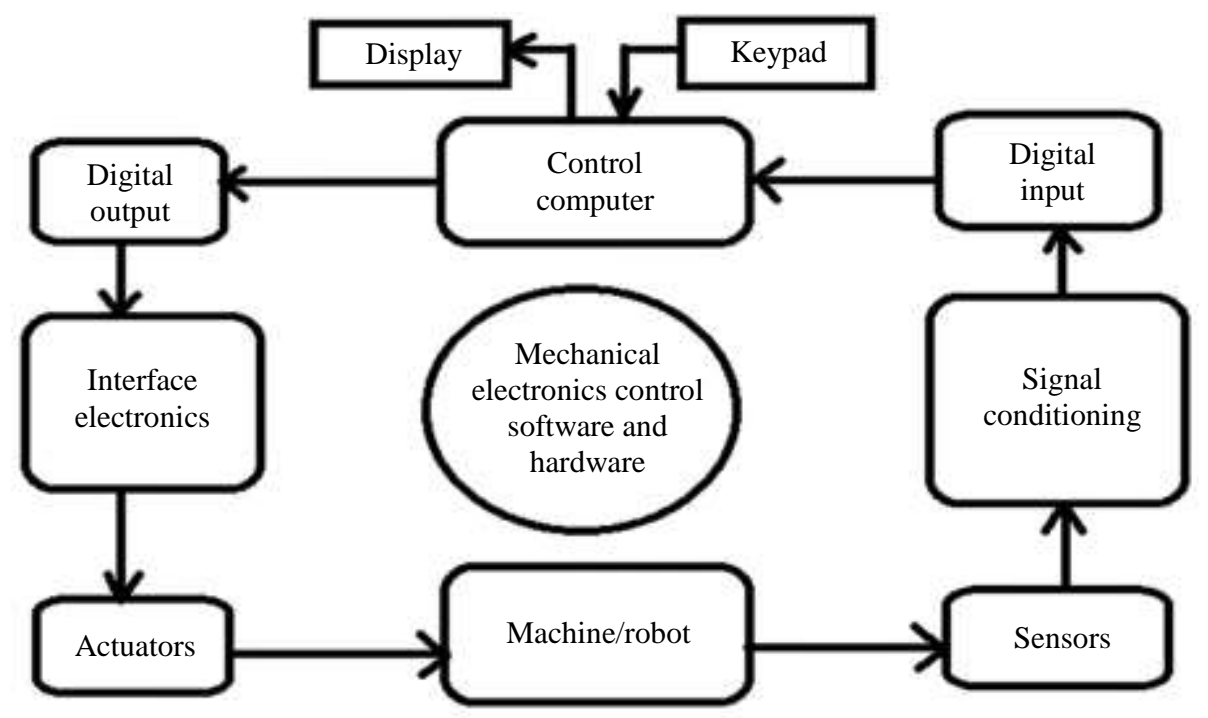

Fig. 2: Key elements of mechatronic system

\section{Applications of Mechatronics}

It will be wondering how mechatronics is implemented in advanced production precisely. Some top examples are here (Dumitru et al., 2015):

a) Production line automation: In a sequence manner, a manufacturing environment is designed whereby one level ends in the next. Throughout the technique, most such systems use belts to move merchandise and substances. Utilizing incorporating devices inclusive of barcode readers, imaging and sound processors alongside the line, mechatronics make it viable to automate the system. As an instance, while on the belt, a packaged product may have its manufacturing and expiry dates stamped in and recorded. (Schweitzer, 1996).

b) Measuring devices: A manufacturing method is most effectively accurate if the final merchandise comes out of inappropriate shapes, sizes, weight and excellent. In this case, it goes an extended manner to make certain the installation of shrewd sensors, testing and calibration structures at the specified points. All of this falls in the mechatronics realm (Schweitzer, 1996).

c) Control systems: In any efficient manufacturing line, measures have to be put in location to make certain that the facility operates at its premiere stage. That makes it a concern to cope with elements like stress and temperature. This will be finished very easily via sensors and reaction mechanisms, way to mechatronics, which works to reveal and hold the desired operating conditions. For example, whilst the temperature grows to a sure diploma, a sensor can cause the cooling machine to show on (Sakai et al., 1999).
The application range of Mechatronics is split into various technical fields in the research and development degree: Motion management, robotics, car systems, shrewd control, actuators and sensors, modeling and design, system integration, manufacturing, micro gadgets and optoelectronics and noise and vibration manage (Dieterle, 2004).

\section{World-Wide Applications of Mechatronics}

The various world-wide applications are: (Craig and Stolfi, 1994):

a) Engineering and manufacturing

b) System

c) Computer-aided and integrated manufacturing systems

d) Transportation and vehicular

e) Structural dynamic systems

f) Systems

g) Medical imaging systems

h) Machine vision

i) Automation and robotics

j) Servo-mechanics

k) Sensing and control systems

l) Automotive engineering

m) automotive equipment in the design of subsystems such as anti-lock braking systems

n) Computer-machine controls, such as CNC, DNC, etc.

o) as computer driven machines like

p) IE CNC milling machines

q) Expert systems

r) Industrial goods

s) Consumer products etc. 


\section{Evolution Level of Mechatronics}

Mechatronics gadget development from a simple mechanical machine is a logical and realistic step inside the improvement of technology and era. Electronics is an important factor that cannot be separated from the mechanical machine.

The mechatronic systems evolution is proven in Fig. 3 (Zambare et al., 2016):

a) Primary Level Mechatronics this level incorporates I/O devices such as sensors and actuators that integrate electrical signals with mechanical action at the basic control levels. Example: Electrically controlled fluid valves and relays. b) Secondary Level Mechatronics this level integrates microelectronics into electrically controlled devices. Example: Cassette players.

c) Tertiary Level Mechatronics this level incorporates advanced feedback functions into control strategy thereby enhancing the quality in terms of sophistication called smart system. The control strategy includes microelectronics, microprocessors and other 'Application Specific Integrated Circuits' (ASIC). Example: Control of Electrical motor used to activate industrial robots, hard disk, $\mathrm{CD}$ drives and automatic washing machines.

d) Quaternary Level Mechatronics this level incorporates intelligent control in the mechatronics system. It introduces intelligence and Fault Detection and Isolation (FDI) capability systems (Yang et al., 2007).

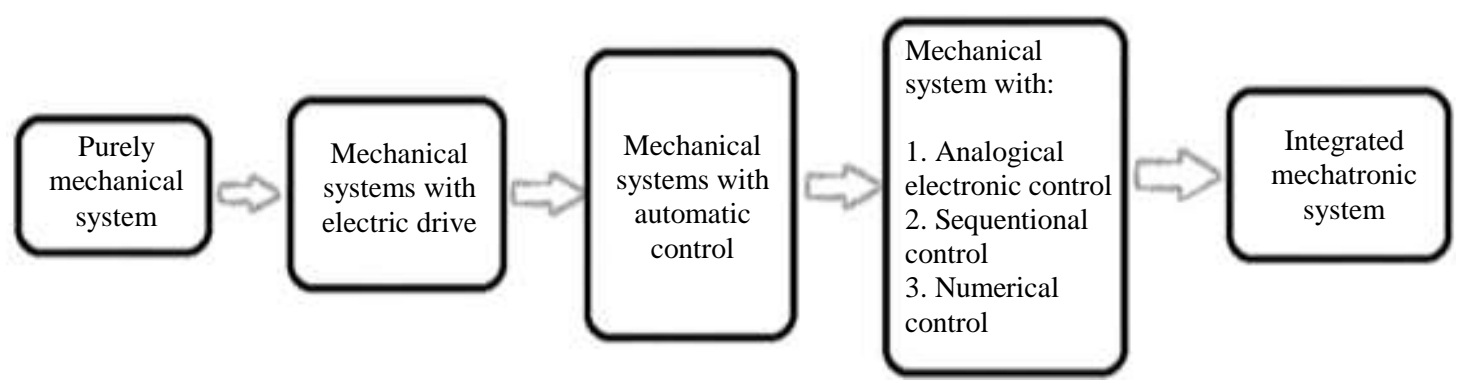

Fig. 3: Evolution of the mechatronics system

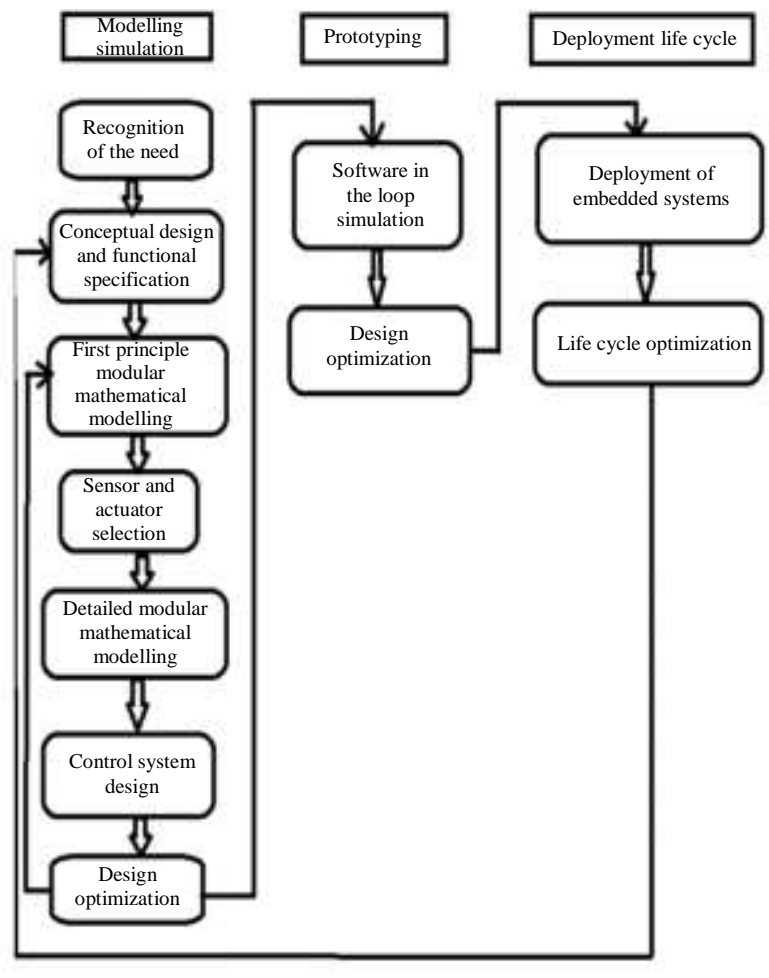

(a)



(b)

Fig. 4: (a) Mechatronics design flow; (b) mechatronics design 


\section{Design Process of Mechatronics System}

Mechatronic format optimizes the parameters at each degree, starting with the primary format and progressing through the manufacturing phase. In offering a coherent framework of thing interactions for gadget assessment, mechatronics utilizes manage structures. Integration inside a mechatronic device is completed with the aid of the manner of mixing hardware (additives) and software (processing of statistics) (Salem and Mahfouz, 2014). The combination of hardware effects from the layout of the mechatronic device is an average machine, bringing the sensors, actuators and microcomputers together into the mechanical device. The integration of software is based mostly on an advanced era. The first step in the focused improvement of mechatronic structures is to examine the patron and the technical environment wherein the device is integrated. The next step is to apply tricky techniques of modeling and outline methods in an incorporated manner to cover all subtasks of the device. There are 3 levels of the mechatronic design manner: Modeling and simulation, prototyping and deployment (Shetty et al., 2012).

Each block is a subsystem that corresponds to certain operations that may be finished bodily or functionally and can be encapsulated right into a block with input/output restrained to enter indicators, parameters and output alerts. This dilemma won't commonly be viable or perfect, of course; however, its use will generate modular subsystem blocks that can be without troubles maintained, exercised, substituted for each other independently (first principle blocks modified with specific blocks and vice versa) and reused in distinct packages.

Considering computer-based totally duties (collectively with records fusion, control and layout testing) rely on a number of the stairs inside the mechatronic layout manner, a powerful pc-aided prototyping environment is crucial (Buur, 1990).

The mechatronic design process is presented in Fig. 4a and 4b. A number of the critical functions of such surroundings are listed and defined underneath:

a) Modeling: Block diagram or visible interface for creating intuitively comprehensible behavioral models of bodily or précis phenomenon. The functionality to encapsulate complexity and hold numerous stages of subsystem complexity is useful.

b) Simulation: Numerical methods for fixing models containing differential, discrete, hybrid, partial and implicit nonlinear (in addition to linear) equations. Need to have a lock for actual-time operation and be capable of executing quicker than real-time.

c) Project Management: Database for preserving assignment facts and subsystem models for eventual reuse. d) Design: Numerical strategies for limited optimization of universal performance talents are primarily based on model parameters and signals.

e) Analysis: Numerical methods for frequency-domain, time-domain and complicated-area design.

f) Real-Time Interface: A plug-in card is used to update a part of the model with real hardware utilizing interfacing to it with actuators and sensors. This is referred to as hardware in the loop simulation or fast prototyping and has to be completed in actual time.

g) Code Generator: Produces green excessive-degree deliver code from the block diagram or visible modeling interface. The managed code can be compiled and used on the embedded processor. The language is usually $\mathrm{C}$.

h) Embedded Processor Interface: The embedded processor is dwelling within the very last product. This option offers communication between the device and the computer-aided prototyping surroundings. This is called an entire tool prototype.

\section{Simulation of Mechatronics Structure}

The simulation modeling of such a device can be solved in the co-simulation of software program MSC.ADAMS and MATLAB etc. (Ciprian et al., 2010).

Using simulations, the designers can test and optimize different parts of the specific system or the entire system using a virtual model or prototype of the part or product on the computers, before actually building it. In the next step before the design process begins simulation results are validated concerning the prototype of the system. This stage can be very difficult and time-consuming but, by simulations, we can reduce the future failure risks and can minimize production costs. Matlab and the dSpace platform can give faster and quicker results.

The mechatronics simulation relation with other systems is shown in Fig. 5.

\section{Mechatronic Destiny Traits}

After figuring out how to harvest, shop and use energy, engineers were capable of conveying mechanics to existence; and as computer technological know-how rose in usefulness, mechatronic engineers began to apply their subject to create clever and complex machines which might be designed to make existence greater comforting and efficient. As these machines end up extra intricate and capable, the destiny of mechatronics will continue to grow and search for approaches we will use strength and mechanics to help accomplish tough or time-ingesting responsibilities, as well as retain to connect the sector and boom our expertise of lifestyles. 


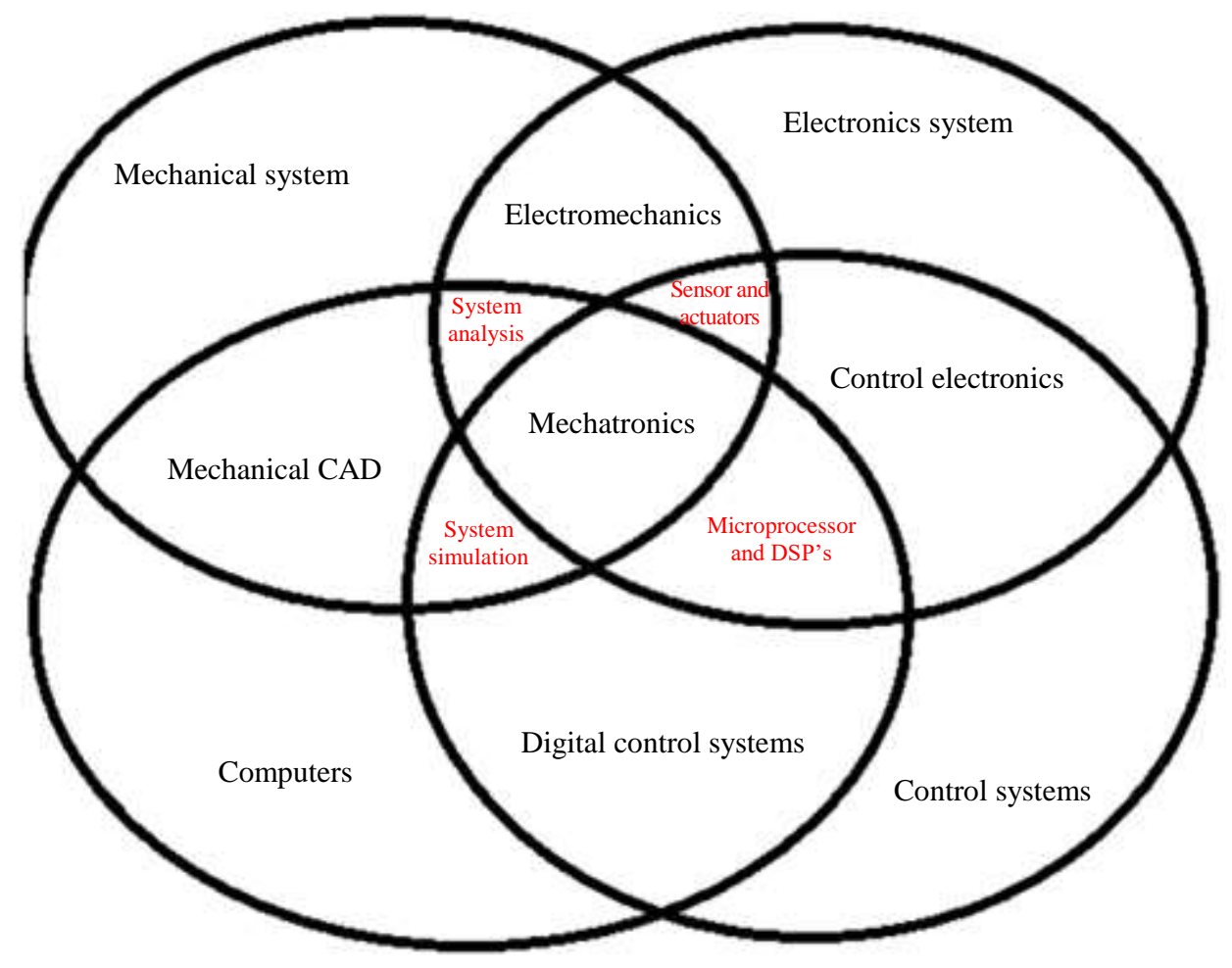

Fig. 5. Mechatronics simulation relationships

\section{Conclusion}

Mechatronics engineering is a new field of engineering that combines mechanical engineering, electronic engineering, software engineering, computer engineering, control engineering as well as system design engineering. Mechatronics ends up an extremely good engineering branch because of its precise function. After 1980 while the computer and era began to develop increasingly more that allowed kind of mechatronics merchandise in distinct lengths which used in every discipline on our lifestyles. Today programs of mechatronics are extremely huge. This period is used anywhere. Robotics is a commonplace instance of mechatronics software program due to terrific development in virtual ships (size, space, performance and so forth.) the ones could take us to the modern-day technology of mechatronics that's called Nano or micro mechatronics.

\section{Acknowledgment}

The research for this paper was supported by research Group of Abhilashi University. We thank Dr. Devinder Sharma,Dean of engineering and management, Abhilashi University; Dr.Jatinder Kaur, Associate professor, Abhilashi University for feedback that considerably extended the manuscript.

\section{Author's Contributions}

Rachita Sharma: Carried out with the graph of work; data collection, evaluation and interpretation and drafting the manuscript.

Bhaskar Dhiman: Participated in conception, vital revision of the manuscript and last approval of the version to be published.

\section{Ethics}

There are no conflicts of interest regarding the publication of this paper.

\section{References}

Buur, J. (1990). Mechatronics in Japan: Strategies and Practice in Product Development. Journal of Engineering Design, 1(4), 327-337.

Ciprian, L. A. P. U. S. A. N., Vistrian, M. A. T. I. E. S., Radu, B. A. L. A. N., \& Olimpiu, H. A. N. C. U. (2010). Modeling and simulation methods for designing mechatronic systems. Journal of engineering Studies and Research, 16, 20-24.

Craig, K. C., \& Stolfi, F. R. (1994). Introduction to mechatronic system design with applications: Course Book. American Society of Mechanical Engineers. 
Diegel, O., Singamneni, S., \& Withell, A. (2008, December). A mechatronics approach to rapid product development: a case study. In 2008 15th International Conference on Mechatronics and Machine Vision in Practice (pp. 588-593). IEEE.

Dieterle, W. (2004). Mechatronic systems: industrial applications and modern design methodologies. IFAC Proceedings Volumes, 37(14), 1-5.

Dumitru, S., Cioboată, D., \& Abălaru, A. (2015). Trends in the development of new intelligent mechatronic equipments with applications in automotive industry. Scientific Bulletin of'Valahia'University. Materials \& Mechanics, 13(10).

Igweonu, E. I., Mbabuike, I. U., \& Okwara, K. K. (2013). Mechatronics-A New Trend In Machine Control.

Milecki, A. (2015, March). 45 Years of MechatronicsHistory and Future. In International Conference on Automation (pp. 127-136). Springer, Cham.

Mishra, P., Ahuja, A., \& Shivpuri, T. (2011). Mechatronics-concept of past core competence of future. International Journal of Power Control Signal and Computation (IJPCSC), 2(2), 88-93.
Sakai, S. I., Sado, H., \& Hori, Y. (1999). Motion control in an electric vehicle with four independently driven in-wheel motors. IEEE/ASME Transactions on mechatronics, 4(1), 9-16.

Salem, F. A., \& Mahfouz, A. A. (2014). Mechatronics Design And Implementation Education-Oriented Methodology; A Proposed Approach. Mechatronics, 1(3).

Schweitzer, G. (1996). Mechatronics-basics, objectives, examples. Proceedings of the Institution of Mechanical Engineers, Part I: Journal of Systems and Control Engineering, 210(1), 1-11.

Shetty, D., Manzione, L., \& Ali, A. (2012). Survey of mechatronic techniques in modern machine design. Journal of Robotics, 2012.

Yang, H., Sun, W., \& Xu, B. (2007). New investigation in energy regeneration of hydraulic elevators. IEEE/ASME transactions on mechatronics, 12(5), 519-526. 